\title{
The Petawatt Laser - Physics Today Article
}

\author{
M. D. Perry
}

July 2, 1997

This is an informal report intended primarily for internal or limited external distribution. The opinions and conclusions stated are those of the author and may or may not be those of the Laboratory.

Work performed under the auspices of the U.S. Department of Energy by the Lawrence Livermore National Laboratory under Contract W-7405-Eng-48. 


\section{DISCLAMER}

This document was prepared as an scoount of work sponsored by an agency of the United States Government. Neither the United States Covernment nor the University of California nor any of their employees, makes any warranty, express or implied, or assumes any legal liability or responstblity for the sccuracy, completeness, or usefulness of any information, apparatus, product, or process disclosed, or represents that its use would not infringe privately owned rights. Reference herein to any specific commerclal product, process, or service by trade name, trademark, manufacturer, or otherwise, does not necessarily constitute or imply its endorsement, recommendation, or favoring by the United States Government or the University of Callfornia. The views and opinions of authors expressed herein do not necessarily state or reflect those of the United States Government or the University of California, and shall not be used for advertising or product endorsement purposes.

This report has been reproduced directly from the best available copy.

Available to DOE and DOE contractors from the Office of Scientific and Technical Information P.O. Box 62, Oak Ridge, TN 37831

Prices available from (615) 576-8401, FTS 626-8401

Available to the public from the

National Technical Information Service

U.S. Department of Commerce

5285 Port Royal Rd.

Springfield, VA 22161 


\title{
The Petawatt Laser - Physics Today Article
}

\author{
Michael D. Perry \\ PO Box 808, L-439, Livermore, CA 94550 \\ Telephone: (510) 423-9234, Fax: (510) 422-5537 \\ e-mail:perrylo@llnl.gov
}

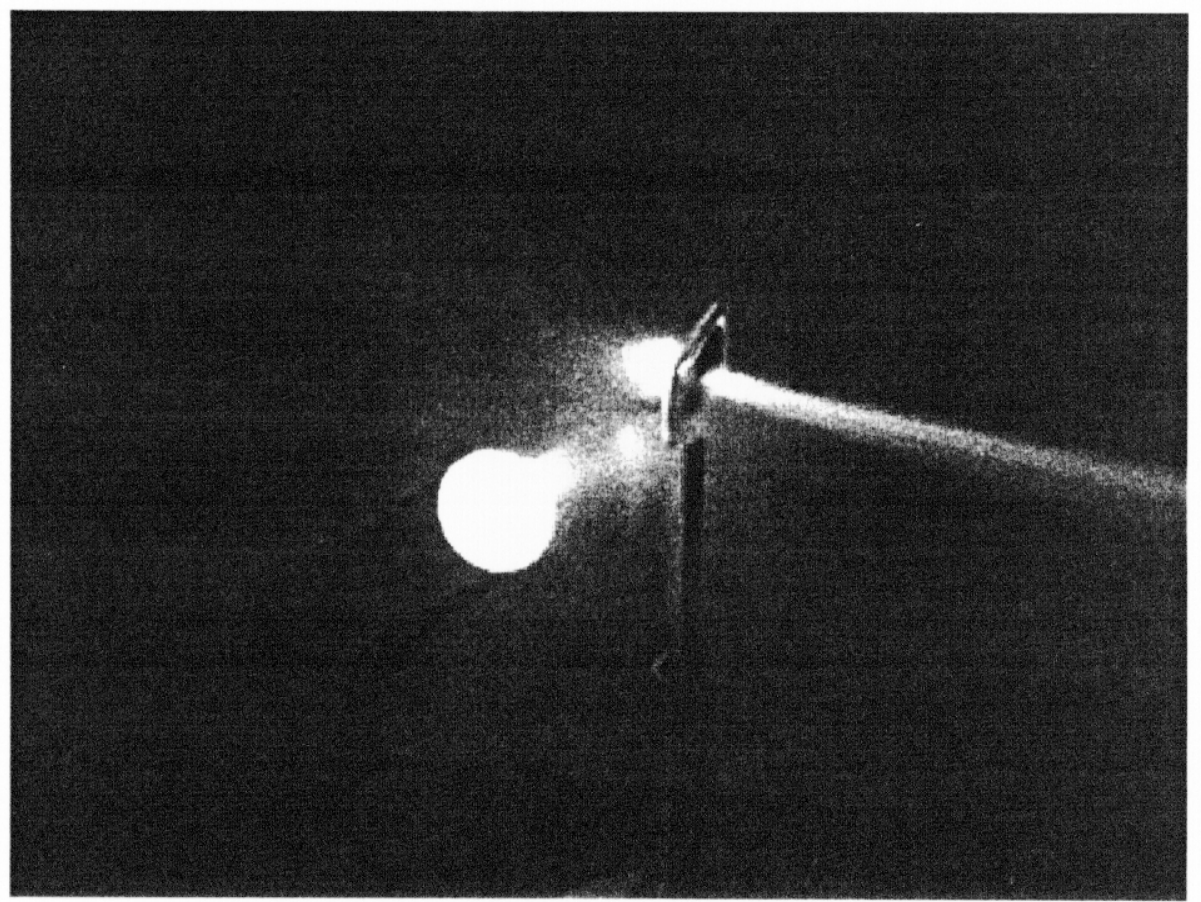

Cover Art: Pholographic image of single beam targel experiment with the Petawatl Laser. The laser pulse is entering from left. The background green light is the result of second harmonic emission from the target. Particles emerging from the back of the target can be observed in a series of jets at right. 
The development of small scale multiterawatt and now petawatt lasers has opened an entirely new regime of laser matter interaction to exploration.[1] Applications of these lasers range from enabling new accelerator concepts[2] to new approaches to inertial confinement fusion (ICF). In 1992, we embarked on a project to develop a laser capable of producing petawatt pulses in order to examine the fast ignitor concept for inertial confinement fusion.[3] Shown schematically in fig. 1, this application requires high pulse energy in addition to the short pulse duration. The essential idea is to pre-implode a deuterium-tritium capsule to an isochoric (uniform density) condition. At the point of maximum compression, irradiate the side of the imploded core with a laser pulse much shorter than the hydrodynamic disassembly time of the irradiated spot $\left(\tau \approx R_{\text {spor }} / v_{s} \leq 10\right.$ psec). Hot electrons $(200 \mathrm{keV}<\mathrm{E}<1 \mathrm{MeV})$ generated by the interaction of the intense $\left(10^{19}\right.$ $10^{21} \mathrm{~W} / \mathrm{cm}^{2}$ ) light with plasma rapidly equilibrate in the dense fuel. The energy equilibration of the electrons raises the overall ion temperature to $5-20 \mathrm{keV}$ initiating fusion burn. High laser pulse energy is required in order to produce enough hot electrons to heat a sufficient number of ions to initiate a self-sustaining fusion burn. In addition, nearly perfect beam quality is required in the laser in order to achieve a small spark region. The Fast Ignitor concept offers the possibility of high target gain at reduced total drive energy than conventional ICF. However, unlike conventional ICF, this approach relies on untested physics in a completely new regime of laser-matter interaction.

To meet the conditions necessary to address the numerous issues associated with the Fast Ignitor and serve as a general facility for experiments in intense laser matterinteraction, the Petawatt laser was designed to produce $1 \mathrm{~kJ}$ pulses with a pulse duration adjustable between 0.4 and $20 \mathrm{ps}$ and sufficient beam quality to produce an irradiance greater than $10^{21} \mathrm{~W} / \mathrm{cm}^{2}$ when focused at $\mathrm{f} / 3$. This design called for a hybrid Ti:sapphire/Nd:Glass laser system in order to provide sufficient bandwidth to achieve the shortest pulse durations, large scale diffraction gratings to compress the pulse after amplification, compression in vacuum in order to avoid beam distortion and self-phase 
modulation resulting from the nonlinear refractive of air and be engineered for routine operation and high levels of reproducibility and reliability. The laser system begins with a standard Kerr-lens mode-locked Ti:sapphire oscillator producing $105 \mathrm{fsec}$ pulses at 1054 $\mathrm{nm}$. A single pulse is selected from the mode-locked train and stretched to $\approx 3 \mathrm{nsec}$ prior to amplification. This pulse is amplified to $6-7 \mathrm{~mJ}$ in a linear Ti:sapphire regenerative amplifier and further amplified to $50 \mathrm{~mJ}$ in a ring-regenerative amplifier. These Ti:sapphire amplifiers are designed to be dynamically stable $\mathrm{TEM}_{\infty}$ cavities with both Kerr and thermal lensing and produce a gain of over $10^{8}$ with no gain narrowing. [4] Further amplification in mixed phosphate glass rod amplifiers produces a spectrally-shaped pulse up to $12 \mathrm{~J}$ in energy.[5]

This pulse is further amplified up to a maximum of $1300 \mathrm{~J}$ by a series of disk amplifiers. Near diffraction-limited beam quality is achieved by limiting the beam to the central $80 \%$ of these amplifiers. Gain narrowing in the phosphate glass amplifiers reduces the spectral width to $4.0 \mathrm{~nm}$ and reduces the pulse duration to $800 \mathrm{psec}$. Additional energy could be extracted from the disk amplifier section but at the price of degraded spatial quality and temporal contrast upon recompression.[6] These effects result from the accumulated nonlinear phase, $\mathbf{B}(\mathbf{r}, \mathbf{t})$,

$$
B(r, t)=\frac{2 \pi}{\lambda} \int n_{2}|E(r, t)|^{2} d z
$$

where the integration is performed over the optical path length through the laser system, $\mathbf{n}_{2}$ is the nonlinear refractive index of the material, and $E(r, t)$ is the spatially and temporally dependent electric field. As an example, a laser pulse propagating through $10 \mathrm{~cm}$ of fused silica at a power density of $10 \mathrm{GW} / \mathrm{cm}^{2}$ accumulates 2 radians of nonlinear phase. The technique of chirped-pulse amplification was developed specifically to overcome the limitations imposed by this nonlinear phase by stretching the pulse prior to amplification thereby reducing the peak power in the amplifiers. However, economic factors dictate that 
petawatt class lasers will almost always be operated at the maximum B-integral allowable in the laser system even with chirped-pulse amplification. A good rule of thumb for most relay imaged laser system is to limit the accumulated nonlinear phase to less than 2 radians. This is a result of the well known degradation of spatial beam quality due to wavefront distortion. Small scale self-focusing causes distortion in the phasefront of the beam which results in decreased focusability or, in severe cases, beam filamentation and damage.

Another effect of the nonlinear phase is self-phase modulation (SPM). This effect is easily observable with ultrashort-pulses by observing the increase in bandwidth of an intense laser pulse upon passage through a nonlinear medium according to,

$$
\omega=\omega_{0}-\frac{\partial B}{\partial t}
$$

Spatial phase modulation is determined by the value of the nonlinear phase while frequency modulation is determined by the time-derivative. For a conventional (near transformlimited) laser pulse with the duration equal to the stretched pulse in a petawatt class laser $(\approx 500-1000 \mathrm{psec})$, the self-phase modulation effect would be negligible. However, the pulse in these system is strongly-chirped (i.e., exhibits a time-dependent frequency). As a result, the central part of the pulse (near the carrier frequency) experiences a greater phase retardation than the early or later part of the pulse where the intensity is less. This limits the ability to recompress the pulse and often results in temporal wings appearing on either side of the compressed pulse.[6]

Following amplification, the chirped pulse is compressed to a pulse duration which can easily be adjusted from 0.43 to $30 \mathrm{ps}$. Pulse compression occurs in vacuum with a pair of large aperture diffraction gratings arranged in a single pass geometry and a compressor throughput of $84 \%$ (figure 2). In most CPA systems the beam ellipticity and spatial chirp associated with a single-pass compressor geometry would be intolerable. However, in the limit that the diameter of the beam is much larger than the dispersed length, these effects are negligible. Currently, the Petawatt operates with a $46 \mathrm{~cm}$ beam and a dispersion length of 
$\mathrm{c} \tau_{\mathrm{str}} * \cos \theta_{\text {compressor }} \approx 20 \mathrm{~cm}$. This system is limited to a maximum pulse energy of 650 due to the use of sub-aperture $(75 \mathrm{~cm})$ diffraction gratings. These gratings exhibit a diffraction efficiency of $95 \%$, a diffracted wavefront quality of better than 0.08 micron (peak to valley) and a damage threshold of $0.42 \mathrm{~J} / \mathrm{cm}^{2}$ for $1054 \mathrm{~nm}$ pulses at $200 \mathrm{fsec}$.[7] Development of gratings of this size, wavefront quality, efficiency and damage threshold was one of the enabling technologies required to achieve petawatt pulses. These gratings will be increased to the full $94 \mathrm{~cm}$ size in July of 1997 and thereby allow operation of the petawatt at $\approx 1 \mathrm{~kJ}$ with a $58 \mathrm{~cm}$ diameter beam.

Most CPA laser systems are designed to produce the minimum pulse duration upon recompression. This is accomplished by setting the compressor to cancel the dispersion of the stretcher and material. Since the compressor cannot cancel the dispersion of the stretcher and material in the laser system exactly, the system is designed to minimize the residual phase, $\delta=\phi_{\operatorname{com}}(\omega)+\left[\phi_{\operatorname{str}}(\omega)+\phi_{\operatorname{mat}}(\mathrm{z}, \omega)\right]=0$. These phase functions are often written in a Taylor series expansion,

$$
\begin{array}{cccc}
\phi_{\operatorname{mat}}(\mathrm{z}, \omega) & \beta_{1}\left(\omega-\omega_{0}\right) \mathrm{z}+ & \underline{\beta_{2}}\left(\omega-\omega_{0}\right)^{2} \mathrm{z}+\underline{\beta} 3\left(\omega-\omega_{0}\right)^{3} \mathrm{z}+\underline{\beta}_{4}\left(\omega-\omega_{0}\right)^{4} \mathrm{z} \\
+\ldots & 2 ! & 3 ! & 4 !
\end{array}
$$

where $\beta n=\left[\partial^{n} / \partial \omega^{n}\right] \omega=\omega_{0}$. The stretcher/compressor combination in the Petawatt laser are designed to correct for chromatic aberration and material dispersion in the system up to third-order (i.e., only the fourth-order terms in $\delta$ remain). This is sufficient for pulses of duration greater than $100 \mathrm{fsec}$. Output pulse characteristics of the petawatt beam under current operational conditions are shown in figure 3 . The fine scale structure in this figure is the result of individual elements of the $\mathrm{CCD}$ array used to record the spectra and autocorrelation.

Target experiments with petawatt pulses are possible either in an independent target chamber or with the Nova laser system for integrated fast ignition. Focusing the beam is 
accomplished using an on-axis parabolic mirror alone, or in conjunction with a secondary "plasma" mirror, as shown in Fig. 4.[8] For irradiances $>10^{14} \mathrm{~W} / \mathrm{cm}^{2}$, short pulse radiation creates a critical density plasma on the surface of a dielectric substrate, with a reflectivity approaching $95 \%$ (fig. 4b). For incident pulses on the order of $500 \mathrm{fs}$, the plasma has insufficient time to undergo hydrodynamic expansion, producing a reflected wavefront comparable to the original optical surface. This novel targeting system enables the production of high contrast pulses, with an easily varied effective focal length as well as off-axis experiments.

Successful completion of the Petawatt laser was enabled by the contributions of many people at LLNL. Particular thanks are owed to J.A. Britten, R. Boyd, C. Brown, S. Herman, J.L. Miller, W. Olsen, D. Pennington, B.W. Shore, B.C. Stuart, G. Tietbohl and $M$. Vergino. 


\section{References:}

1. M.D. Perry, and Gerard Mourou, "Terawatt to Petawatt Subpicosecond Lasers," Science, 264, 917 (1994).

2. C. Joshi and P.B. Corkum, Physics Today, January 1996.

3. M. Tabak, J. Hammer, M. E. Glinsky, W. L. Kruer, S. C. Wilks, J. Woodworth, E. M.Campbell, M. D. Perry, and R. J. Mason, "Ignition and high gain with ultrapowerful lasers," Phys. Plasmas, 1, pp. 1626-1634, 1994.

4. B. Stuart, S. Herman and M.D. Perry, "Broadband Amplification in Ti:Sapphire beyond $1 \mu \mathrm{m}$," IEEE J. Quan. Elec., 31, 528 (1995).

5. M. D. Perry, F. G. Patterson, and J. Weston, "Spectral Shaping in Chirped-Pulse Amplification," Optics Letters, 15, 381 (1990).

6. M.D. Perry, T. Ditmire, and B.C. Stuart, "Self-Phase Modulation in Chirped Pulse Amplification," Optics Letters, 19, 2149 (1994).

7. R. Boyd, J.A. Britten, B.W. Shore, B. Stuart, and M.D. Perry, "High Efficiency Metallic Gratings for Laser Applications," Applied Optics, 341697 (1995).

8. M. D. Perry, V. Yanovsky, M. Feit, and A. Rubenchik, "Plasma mirrors," Phys. Plasmas, submitted, 1997. 


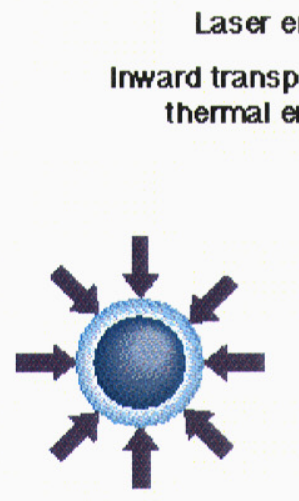

Atmosphere

Formation

Laser or particle beams rapidly heat the surface of the fusion target forming a surrounding plasma enrelope
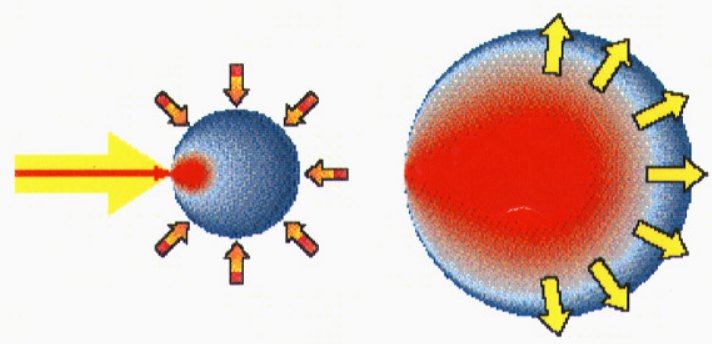

Ignition

At the moment of maximum compression a short (1-10 psec) high intensity $\left(10^{19} \mathrm{mcm}^{2}\right)$ pulse ignites the capsule
Burn

Thermonuclear burn spreads rapidly through the compressed fuel, yielding many times the driver input energy
Fuel is compressed by rocket-Iike blowoff of the surface material

Figure 1: Diagram of Fast Ignition concept in inertial confinement fusion 


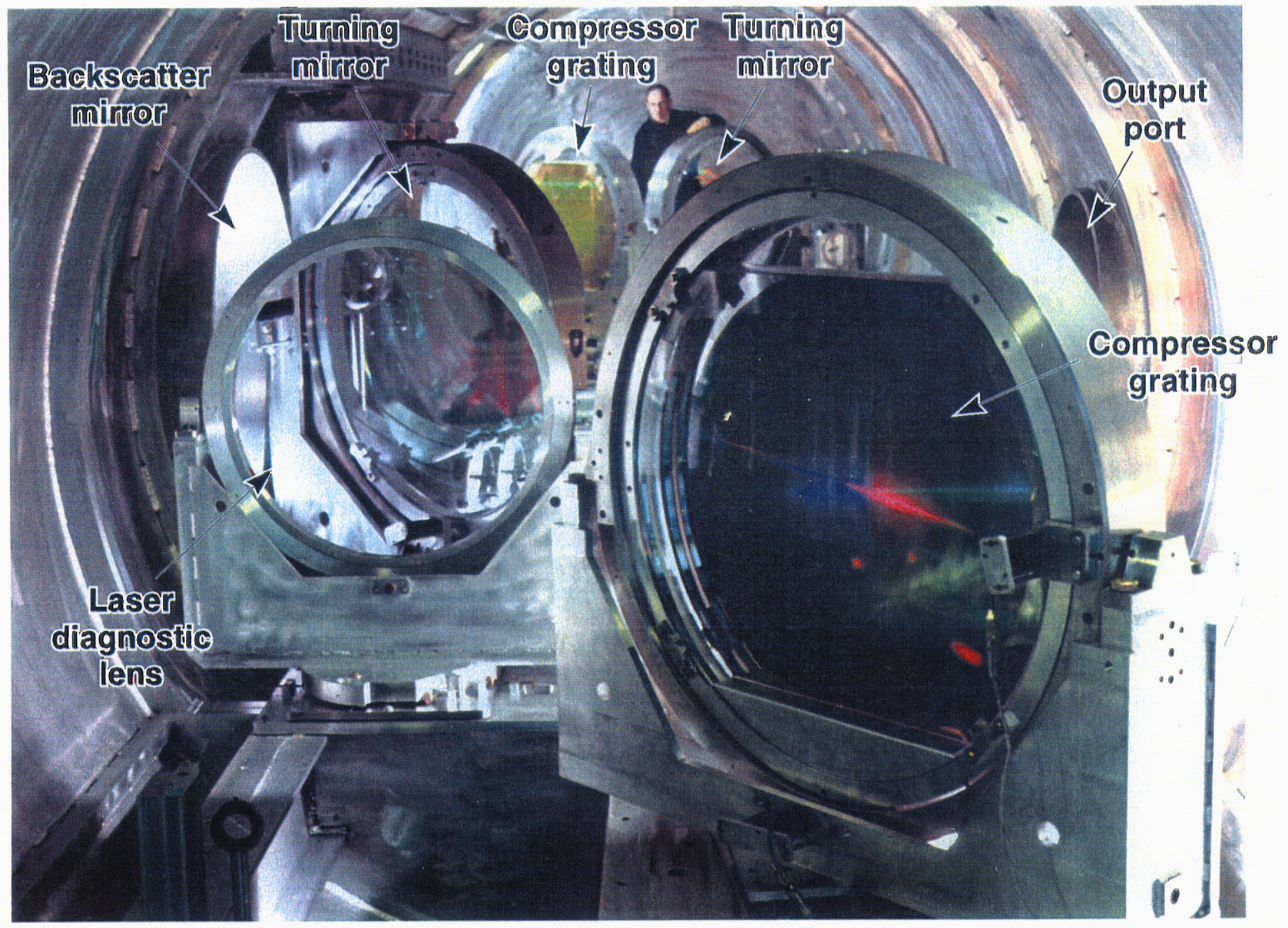

Figure 2: View inside the Petawatt Compressor Chamber 

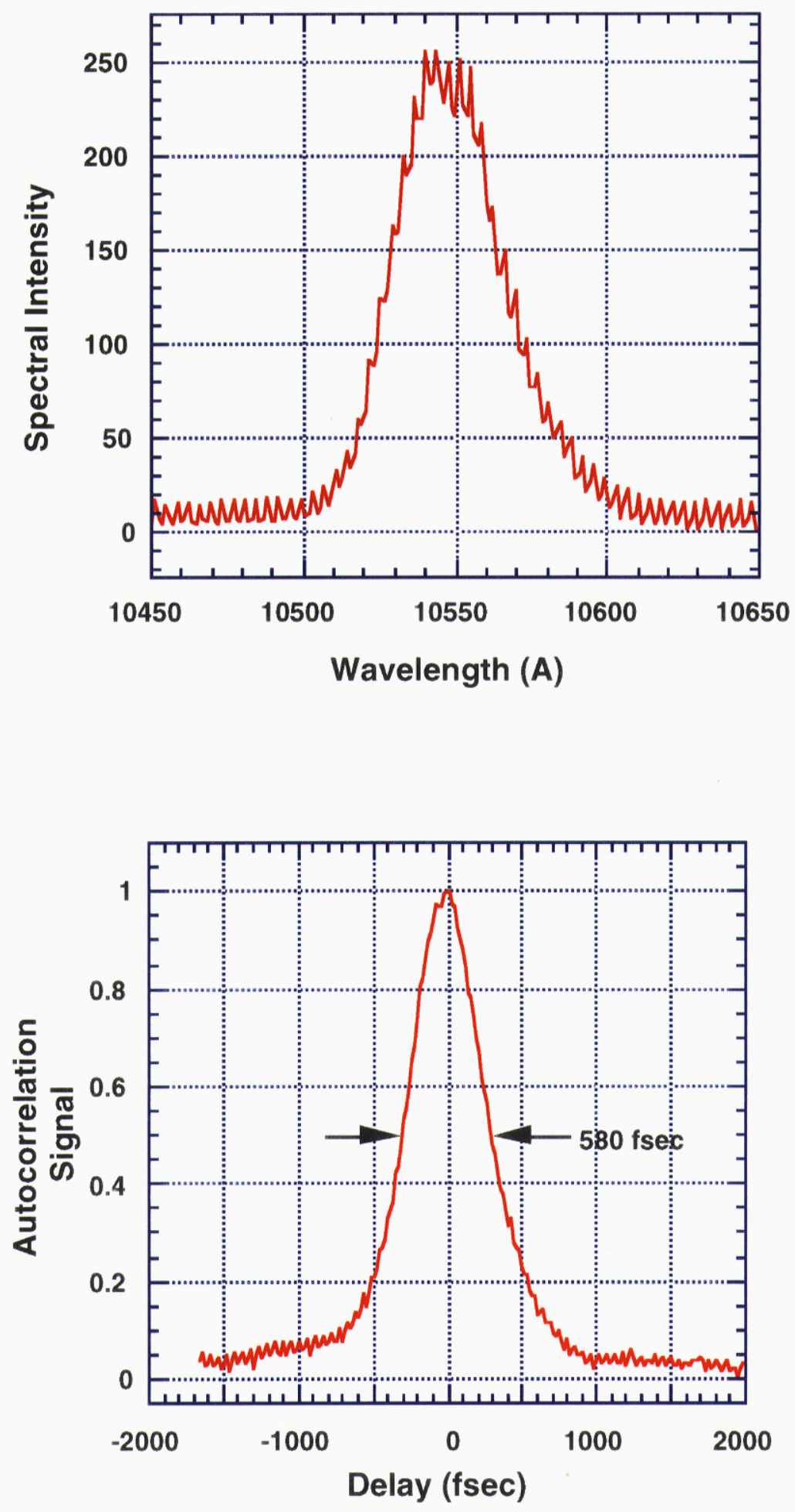

Figure 3: Measured pulse parameters in the first Petawatt shot series (May 1996) at an energy of $620 \mathrm{~J}$. a) Near-field beam profile, b) pulse spectrum and c) autocorrelation (deconvolved pulsewidth $=420 \mathrm{fsec}$ ), $\Delta v \Delta \tau=0.43$. 
a)

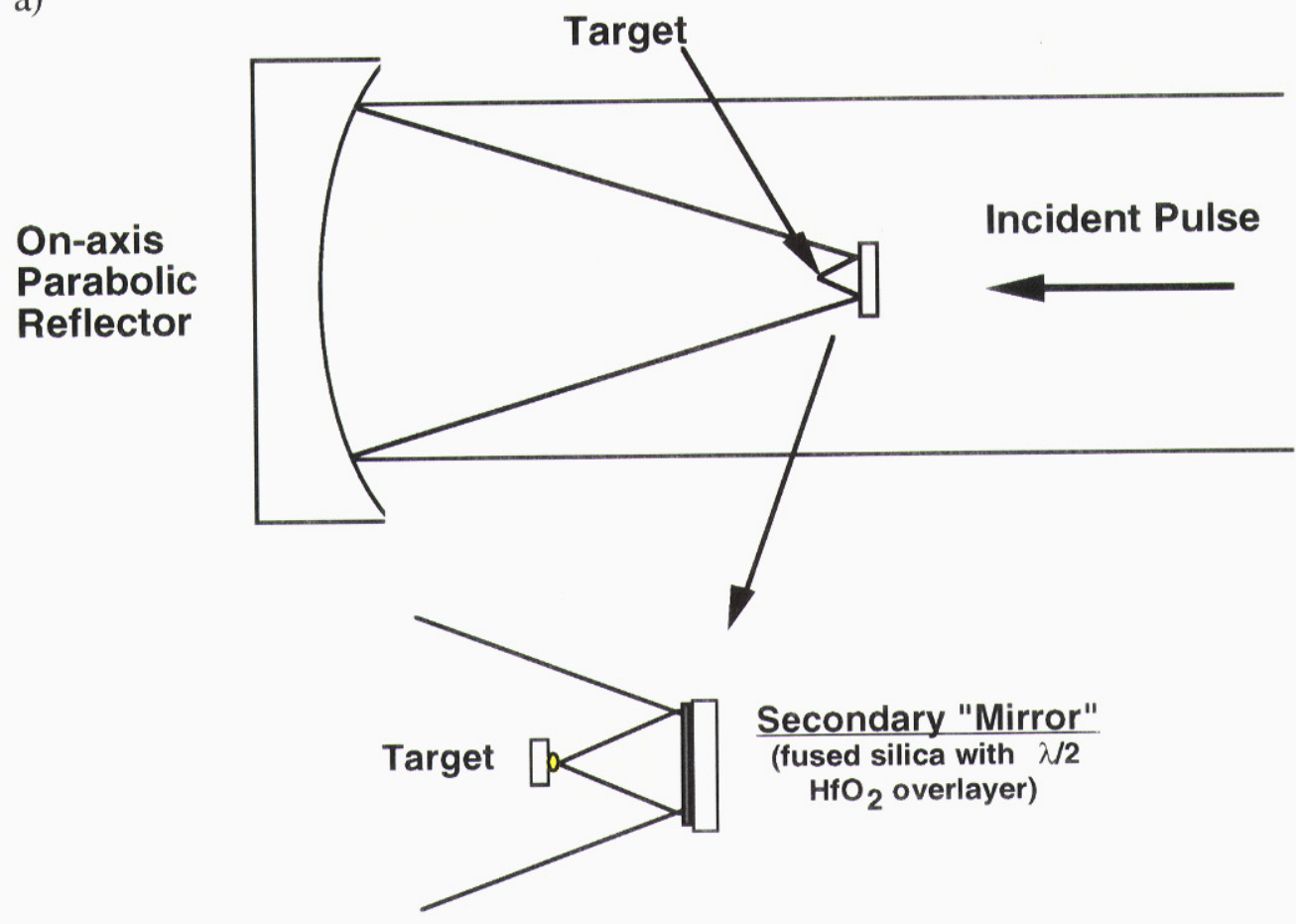

b)

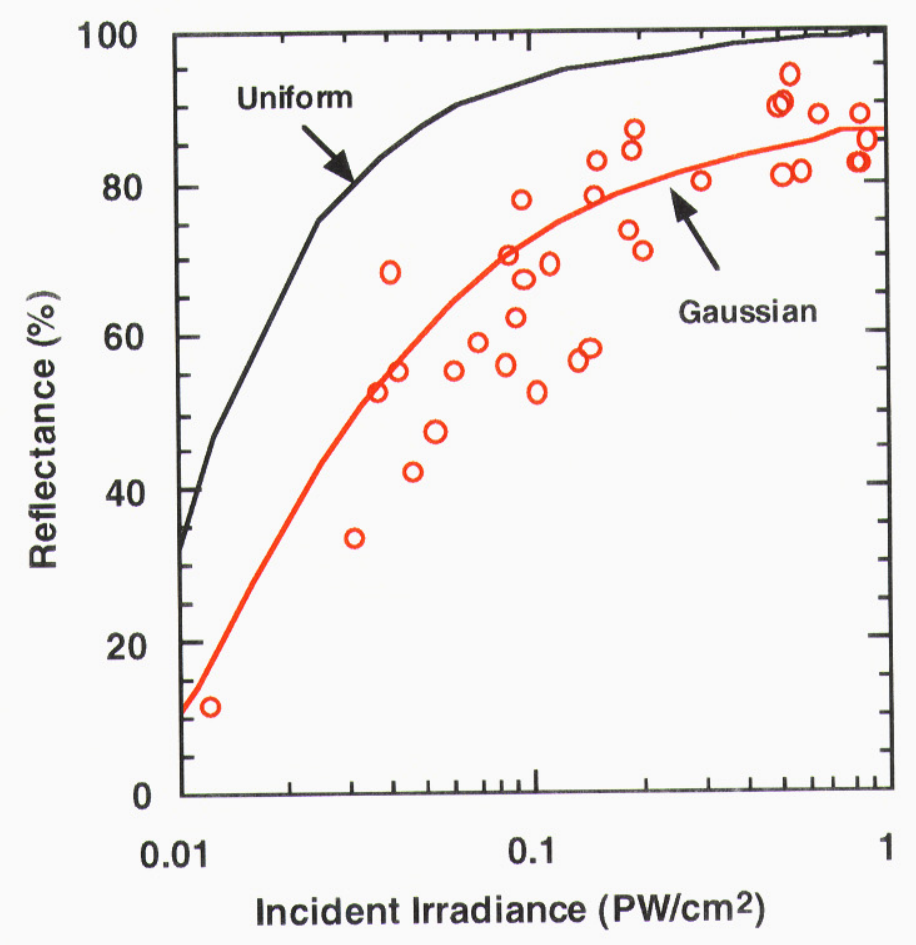

Fig. 4: a) Cassegranian focusing concept using a plasma for the secondary mirror.

b) Measured reflectivity from the plasma mirror 


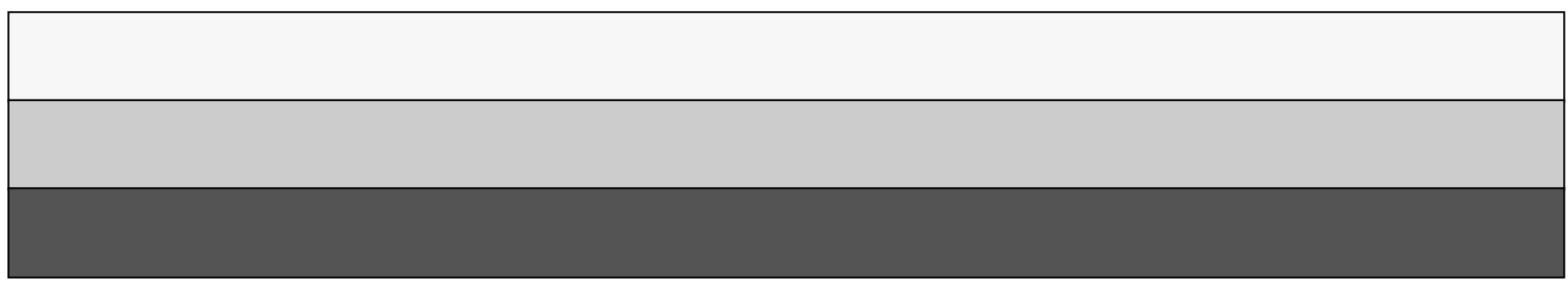

